\title{
Alfabetismos transmedia: cultura maker y aprendizajes colaborativos en el mundo hiperconectado
}

José Manuel Corona Rodríguez*

DOI: https://doi.org/10.17230/9789587206289ch8

\section{Introducción}

El empoderamiento de las comunidades makers ha cobrado cada vez más visibilidad, importancia y seguidores a lo largo del mundo, popularizándose con el desarrollo de la impresión 3D, los drones, el desarrollo de software y hardware, la robótica, y en general el DIY (Do it Yourself). En este trabajo hago una reflexión sobre el contexto comunicativo de las estrategias que adoptan y ejecutan los miembros de la comunidad MakersGDL, y cómo estas se relacionan con el desarrollo de aptitudes y habilidades para la implementación de un currículo de alfabetismos transmedia desde una perspectiva constructivista de la educación, basado en la participación activa de sus miembros en un entorno transmediático.

De este modo, partimos del potencial creativo y productivo con el que cuentan los individuos contemporáneos para participar en la generación de contenidos mediáticos y objetos físicos tangibles a partir de la reconfiguración de los medios de producción, las transformaciones en las lógicas tradicionales de distribución (y validación) y el constante acceso a más información, lo que desencadena la producción de nuevo conocimiento. En este contexto cobra cada vez más relevancia el concepto propuesto por Castells (2010) de la autocomunicación de masas, con el cual se describe la capacidad de los medios interactivos para facilitar la creación de contenidos que llegan a grandes audiencias.

A su vez, la cultura de la participación resulta muy útil a partir de la intersección de tres perspectivas. Primero, las nuevas tecnologías de la comunicación, que permiten a los consumidores apropiar y recircular el contenido mediático a su disposición. Segundo, el amplio grupo de comunidades que promueve el Do It Yourself (DIY o Hágalo usted mismo)

Licenciado en Comunicación de la Universidad Autónoma de Puebla y magíster en Comunicación de la Universidad de Guadalajara. Estudiante de Doctorado en Educación y profesor de la Universidad de Guadalajara, México. Correo electrónico: jmanuel.corona@academicos.udg.mx 
para la producción mediática, informativa, de software y de objetos de muchos tipos. Tercero, las tendencias económicas que soportan el conglomerado de medios propicia el flujo de imágenes, ideas y narrativas a través de múltiples medios, lo cual demanda una mayor participación de las personas (Jenkins, 2008). Así, abordaremos aquí las prácticas DIY como un conjunto de valores y actitudes que alientan la creación individual y colectiva de contenidos mediáticos y de productos tangibles con base tecnológica, lo cual se traduce en un desarrollo constante de los alfabetismos transmediales.

Indagar en los procesos de participación significa hacer hincapié en las prácticas comunicativas colectivas, lo cual supone una exploración que se preocupe por las formas, los niveles y los tipos de participación. Tal como lo plantean Ferrés y Piscitelli (2012, p. 13) la participación no debe limitarse a la mera posibilidad de la expresión, ya que "los procesos de análisis de mensajes han de ser afrontados también desde un planteamiento activo, dialógico, tomando en consideración la participación del interlocutor mediante los procesos de selección, interpretación, aceptación o rechazo, crítica, difusión, etcétera”. Esto implica una reformulación de los principios con los cuales se entiende y se reflexiona sobre la agencia de los individuos.

En este escenario se destaca la dimensión creativa e innovadora de la participación a partir de espacios y contenidos de afinidad que pueden considerarse espacios de aprendizaje informal colectivos y colaborativos alrededor de un interés compartido, una temática, una narrativa o una ética (como la ética hacker) (Roig, 2011). La cultura de la participación tiene una base sustancial en la dinámica de la sociedad informacional caracterizada por un incremento de la capacidad (y acaso también voluntad) de los usuarios en relación con los procesos de producción y consumo cultural. Esta dinámica se manifiesta en la producción personal y colectiva de contenidos y productos, que según Jenkins (2010) ha adquirido una dimensión sin precedentes a través de la internet y otras tecnologías asociadas.

Acceder a la cultura de la participación supone nuevas formas de currículo oculto, que se está configurando a partir de los vínculos con los medios y sus contenidos y en particular con los contenidos que logren crear engagement (compromiso) basado en la dimensión emotiva y afectiva (de ahí la importancia de los discursos que promueven el DIY). En este contexto se han desarrollado un conjunto de nuevas habilidades mediáti- 
cas que los sujetos necesitan para participar activamente en el escenario convergente de la cultura. Según Livingstone (2008), la cultura de la participación mueve el foco de la educación-alfabetización individual a la expresión y ejecución de habilidades a través del involucramiento en comunidades de práctica.

En este contexto, investigar la transmedialidad implica revisar el modelo teórico que sustenta la idea de la convergencia cultural, desde el cual se propone que los actores sociales se relacionan e interactúan con los textos y contenidos mediáticos de formas más inmersivas y significativas (Ibrus \& Ojamaa, 2014). Hablar de transmedialidad obliga a reconocer una tensión entre la participación creativa y la reactiva, que según Orozco (2011) es la más usual (la reactiva), y que se describe como parte de una condición comunicacional contemporánea en la cual la creciente ubicuidad de las audiencias y la hiperconectividad han reforzado la impresión de que el consumo mediático se ha vuelto productivo al quedar al control de las audiencias.

Las reflexiones de Orozco (2011) son pertinentes ya que por un lado invitan a reconocer los cambios en las formas de ser audiencias, pero sin dejar de ser críticos sobre las formas de participación real (o efectivas) que se están propiciando y generando en los entornos comunicativos. La condición comunicacional a la que se refiere este autor implica permitir a las audiencias asumirse activas más allá de la mera reinterpretación o la deconstrucción simbólica. El tránsito de ser receptor a ser productor supone uno de los cambios sociales más significativos, al propiciar una cultura real de la participación. Asumir estos cambios en las maneras en que ocurre la comunicación implica apoderarse de la premisa epistemológica que supone investigar a los actores de la comunicación como agentes activos.

Scolari (2008) relaciona los cambios en los roles de los actores comunicativos en términos de una creciente transmedialidad que desdibuja las fronteras entre los medios de comunicación y sus formas de operación. En este sentido la transmedialidad implica un cruce transversal y una combinación de los lenguajes y formas de operación entre los viejos medios y los nuevos. En este sentido la investigación alrededor de este concepto se ha caracterizado por analizar las maneras en que los contenidos fluyen en una red articulada de textualidades, medios y plataformas. 


\section{De la alfabetización a los alfabetismos transmediales}

La exploración de los usos educativos de las lógicas transmedia se ha convertido en un tema frecuente en las discusiones académicas en años recientes. Con el objetivo primordial de potenciar el efecto de producir una experiencia de inmersión y engagement que multiplique el número de oportunidades de aprendizaje a partir de la generación de experiencias basadas en la interactividad y la participación, o con la idea de potenciar la participación y creación de contenidos por parte de los estudiantes.

Durante la última década, en el contexto de un mundo altamente mediatizado, el concepto de transmedia se ha posicionado con fuerza para reflexionar sobre las relaciones entre las audiencias, los productores y los contenidos. Acuñado inicialmente por Marsha Kinder (1980), el concepto de transmedia se refiere a un conjunto de elementos narrativos y no narrativos que se extienden a través de múltiples plataformas tecnológicas y de medios. Según Dena (2009), lo transmedia toma diferentes formas dependiendo del contexto, las audiencias y los propósitos, las cuales al emplearse y combinarse dan como resultado una transmedia practice.

Por su parte, la propuesta de Alper y Herr-Stephenson (2013) consiste en desarrollar una transmedia play que radica en la experimentación y la participación de experiencias transmedia por parte de los estudiantes. Usualmente, las lógicas transmedia operan en relación con estructuras contemporáneas del entretenimiento y el marketing, y las aplicaciones posibles a la educación son cada vez más investigadas y puestas en práctica.

En este sentido, se hace evidente que las lógicas transmedia se basan en las habilidades de las audiencias para decodificar, remezclar, crear y distribuir diferentes tipos de contenidos y narrativas de los medios. El valor de la agencia de los individuos para el aprendizaje es algo que cada vez más se apunta como necesario para investigar desde numerosas perspectivas. Para Alper y Herr-Stephenson (2013) la idea de una transmedia play centrada en la alfabetización responde por lo menos a dos de los principios fundamentales promovidos por la National Education Association for Media Literacy; el primero sugiere que se debe promover un consumo crítico y activo de los medios, situación que ocurriría con mayor frecuencia en un enfoque transmedia al motivar a las audiencias no solo a desarrollar un sentido crítico sobre los mensajes sino además al involucrarse activamente en la construcción de significados y contenidos. El segundo se refiere a 
que la alfabetización mediática expande en su sentido el concepto mismo de alfabetización, razón por la cual un enfoque transmedial de los alfabetismos expandiría aún más este concepto al facilitar nuevas o diferentes formas de lectura, escritura, autoría, participación y creación.

Hay múltiples investigaciones que tienen como objetivo contribuir al desarrollo y aplicación de las narrativas transmedia a entornos educativos a través de marcos y modelos educativos basados en aprendizajes centrados en experiencias inmersivas (Rodriguez y Bidarra, 2014). En última instancia, la mayoría de las investigaciones de este enfoque se proponen demostrar cómo la creación de un ambiente transmedia de aprendizaje permitiría al alumno desarrollar y prosperar en los ambientes digitales. Esta visión advierte claramente una vinculación entre la alfabetización tradicional y una alfabetización pensada en la lógica transmedia.

En investigaciones como la realizada por Raybourn (2014) se sugiere la necesidad de crear experiencias de aprendizaje transmedia a partir del diseño de métodos basados en la simulación e interactividad propia de los videojuegos. Su propuesta está centrada en identificar y abordar la interacción, la narrativa, la cultura emergente y los lugares virtuales en los que ocurren las experiencias. Esta estrategia con finalidades de alfabetización consiste en el diseño de una campaña o sistema de juegos que integre personajes, roles, tareas, interfaces y redes sociales virtuales.

En este sentido, si bien la transmedialidad y sus lógicas de operación pueden ser extrapoladas a ciertas prácticas educativas a partir de diseños instruccionales, los procesos para que esto ocurra no pueden ser pensados en el vacío, y es necesario establecer suficientes matices sobre los sujetos participantes, tanto aprendices como maestros (si es que acaso esas entidades siguen siendo válidas).

Son tres las principales debilidades y deficiencias sobre los proyectos centrados en implementar o incorporar las lógicas transmedia a las prácticas educativas: primero, que suelen estar exageradamente centrados en la tecnología y los medios, otorgándole a esta poderes y facultades que no necesariamente posee; segundo, desnaturalizan (en el sentido de que se derivan de una estrategia) el consumo y la participación; y tercero, ignoran y pasan por alto que los contenidos transmediales tienen una condición centrada en el compromiso (engagement) de los sujetos a partir de lo emotivo y lúdico más que por una tarea impuesta. 
A pesar de lo anterior, es necesario reconocer que estos esfuerzos denotan un propósito para desarrollar en los estudiantes algunos de los mismos objetivos, habilidades y competencias que la alfabetización mediática ha perseguido históricamente. La diferencia consistiría en los métodos empleados -y en la comprensión de lo que en realidad significa la transmediación, no solo en términos de los medios de comunicación, sino principalmente en la agencia de los sujetos- para realizar esta alfabetización transmedia. Si se acepta que el escenario de la comunicación es (más que nunca) convergente, no puede entonces pensarse en términos únicamente de los medios masivos de comunicación. En virtud de que, por ejemplo, ver televisión en nuestros días significa poner en juego diferentes vínculos con los contenidos y las maneras en cómo nos relacionamos con otros sujetos similares a nosotros y con los relatos, históricamente pensados para las audiencias (Orozco, 2014).

Se observa aquí una paradoja que resulta muy problemática: por un lado la investigación sobre las alfabetizaciones (mediáticas, informacionales, digitales, de internet, etc.) es la principal puerta de entrada para investigar desde una mirada educativa los entornos comunicativos en los que se desenvuelven los sujetos, pero, por otro lado, en el afán de incorporar los mismos modelos que se han desarrollado a lo largo de las investigaciones sobre la alfabetización mediática, se corre el riesgo de invisibilizar y simplificar las prácticas de los sujetos.

Con esta misma lógica, Wenger, McDermott, \& Snyder (2002) señalan que el aprendizaje en sí mismo no se puede diseñar, lo que se diseña son infraestructuras sociales que pueden fomentar ciertos procesos de aprendizaje; esto supone que siempre existe "una incertidumbre intrínseca entre el diseño y su realización en la práctica, porque la práctica no es el resultado del diseño, sino de los sujetos inmersos en ellas y sus disposiciones, limitaciones, capacidades, deseos, voluntades y un largo etcétera que escapa a las previsiones de los diseñadores o hacedores de estrategias de aprendizaje" (2002, p. 12). ${ }^{1}$ Por esta razón no se trata simplemente de adaptar o diseñar los escenarios transmediales para fomentar el aprendizaje, más bien son las prácticas sociales y culturales las que lo hacen posible.

${ }^{1}$ Las traducciones de los textos citados son mías. 
Como lo hemos planteado hasta aquí, las prácticas que resultan de la convergencia cultural -centradas en las culturas de la participación y la colaboración- presentan complejos desafíos y retos para los investigadores y educadores al momento de desarrollar estrategias capaces de superar las dualidades y limitaciones de modelos anteriores. La idea de los alfabetismos transmediales que propongo aquí busca superar una postura centrada en la crítica a los medios y la reproducción de tareas escolares basadas en los modelos tradicionales de la comunicación y la educación (como lo ha hecho la alfabetización mediática tradicional). La propuesta de investigación más cercana y que dialoga de una forma directa con los planteamientos y preguntas que me he formulado en esta propuesta es la que realiza Scolari (2016), al proponer un alfabetismo transmedia que sea capaz de desplazarse de la enseñanza formal hacia el aprendizaje informal.

Alfabetizar implica un proceso que se define por la planificación y la voluntad explícita de enseñar (a leer y escribir primero y luego a producir contenidos mediáticos). Como se sabe, el concepto de alfabetización proviene de la palabra literacy en inglés; no obstante, en las investigaciones más recientes se percibe una transformación que va de la alfabetización a los alfabetismos, o new literacies (Gee, 2015; Pérez, 2014). Esta transformación implica superar la idea de la alfabetización como un proceso escolarizado, planificado e institucional para darle cabida a un proceso donde las prácticas sociales y culturales definen el tipo de alfabetismos que se están dando en los ambientes extraescolares.

Lankshear \& Knobel (2006) proponen pasar de la alfabetización a los alfabetismos porque afirman que a través de estos es posible abarcar la complejidad de las prácticas culturales que propician los aprendizajes, más allá de si son planificados o no. En este sentido el alfabetismo es visto como una práctica social que no depende de condicionamientos formales o planificados. Por esta razón propongo el uso de alfabetismos y no la mera alfabetización.

Para Dussel (2010) es preferible usar alfabetismos en lugar de alfabetización porque el concepto de alfabetización implica una connotación directa a las prácticas de lectura y escritura que no abordan completamente los contextos y prácticas en las que leer y escribir ocurren. Como se ha visto, con la creación de una amplia variedad de conceptos que tratan de abordar las nuevas alfabetizaciones lo que se hace es expandir la metáfora de la lectura y la escritura a otros contextos sin lograr atender a 
la complejidad de las prácticas sociales y culturales que ocurren más allá de la voluntad por enseñar o aprender.

\section{Colaboración Do it Yourself de hackers y makers}

El movimiento Hazlo tú mismo, o Do it Yourself (DIY), ha significado un renovado interés por la creación y fabricación propia y ha posibilitado una reivindicación de las posibilidades expresivas de los individuos en función de los contenidos mediáticos, como resultado de una alternativa a la desintermediación en la producción de contenidos, materiales, ideas o tecnología, antes solo en posesión de corporaciones o empresas. En términos de las comunidades, Jenkins (2010) sugiere que el hazlo tú mismo no significa "hazlo tú solo", sino que, por el contrario, el componente social y colaborativo es fundamental para suscitar experiencias significativas de aprendizaje y de transferencia de conocimiento. La importancia del DIY para la investigación de las culturas de la participación implica mirar con detenimiento prácticas de producción mediática (pero no solo mediática sino también de hardware y software) que suponen una reformulación de las maneras en que se aprende colectiva y colaborativamente, en función de intereses de ocio, emprendimiento, innovación y/o cívicos.

El Do it Yourself se puede entender como las prácticas de participación de las personas para producir contenido propio, en función de discursos mediáticos o a partir de información disponible en los medios. Este producir contenido propio significa la creación de una amplia variedad de composiciones textuales, sonoras, visuales, etcétera, pero también significa la intervención, modificación o creación de software, hardware, objetos, procesos o tecnología especializada.

La investigación del DIY está asociada usualmente a la ética y cultura hacker, que centra su actividad en la innovación por medio de la apropiación, intervención y creación de sistemas, productos, y procesos culturales y tecnológicos (Libow \& Stager, 2013). La figura del hacker tiene una doble connotación; una negativa, asociada a la capacidad de las personas para utilizar conocimiento especializado informático destinado a vulnerar la seguridad de los sistemas computacionales, de servicios digitales y empresas o corporaciones virtuales. Y una connotación positiva asociada a la puesta en práctica de conocimiento especializado para reconfigurar sistemas y prácticas en beneficio de la sociedad a partir de la apertura 
de la información y el conocimiento para la gente a través de estructuras abiertas y horizontales, todo en beneficio de lo comunitario (Himanen, 2011).

La cultura y la ética hacker, en relación con la educación, se ha conectado sobre todo a partir del estudio del software libre. Estos trabajos se han concentrado especialmente en la realización de etnografías que muestran una descripción detallada de las prácticas fundamentales que se centran en la programación y la creación de código abierto. La figura del hacker se ha presentado como una alternativa de transformación política, económica y tecnológica que gana cada vez más presencia en la cultura y en los discursos mediáticos (Estalella, s. f.).

El trabajo etnográfico que se ha realizado sobre la cultura hacker y el movimiento DIY se ha propuesto la descripción de las prácticas a partir de la identidad de los sujetos en relación con las comunidades a las que se integran según los intereses particulares y sus prácticas comunicativas. Estas perspectivas de investigación ayudan a pensar no solo los rasgos de la identidad de los sujetos sino además a poner en la agenda la reflexión sobre la colaboración, la participación, lo colectivo, la transmedialidad y principalmente sobre las nuevas maneras en que el aprendizaje está ocurriendo en comunidades de práctica basadas en la cultura hacker y de emprendimiento.

El movimiento maker se puede entender en ese sentido como heredero de la tradición de la cultura hacker en cuanto comparte los ideales cívicos y es capaz de participar de una forma intensa sobre las manifestaciones culturales y problemas sociales que tiene a disposición. El movimiento maker tiene como antecedente directo el DIY que capitaliza múltiples maneras de creación colaborativa, y que responde (entre otros problemas) a la obsolescencia programada mediante la participación basada en la intervención de los procesos de producción y circulación de productos.

La investigación social sobre los makers (en las ciencias sociales) tiene una fuerte vinculación antropológica en cuanto busca comprender las prácticas de los sujetos en función de su acción colectiva y con base en una desterritorialización constante que va de lo online a lo offline y viceversa. El movimiento maker (o de los hacedores) opera con una lógica y ética establecidas en un texto fundamental y fundacional para todos los miembros de estas comunidades. Se trata del libro de Cris Anderson (2012) Makers. La nueva revolución industrial en el que se auguró que las fábricas 
volverán a los hogares, anticipando el continuo proceso de desintermediación que ha surgido en los últimos años en la elaboración de productos. El argumento central de este texto es que, así como la internet modificó el flujo de la información, los contenidos y la comunicación, el software de última generación, las impresoras 3D y otras máquinas para fabricar objetos cambiarán los procesos de participación y creación, basándose en dinámicas de colaboración y creatividad al interior de comunidades de afinidad.

La comunidad de makers tiene presencia internacional a través de redes sociales digitales y se caracteriza también por la concreción de sus prácticas en los makerspaces, que son en realidad espacios dedicados para la creación colectiva y la socialización de los miembros (Hatch, 2014). El maker trasciende al aficionado en cuanto logra establecer vínculos comunitarios, empresariales, educativos, económicos y políticos con otras comunidades y agentes relevantes. Y especialmente se puede distinguir al maker de otros grupos similares, no solo por lo anterior sino además por su disposición técnica para aprender a usar maquinarias y dispositivos para la consecución de sus objetivos.

Es indispensable entender que la investigación sobre los makers implica un vínculo indisoluble con el movimiento DIY que a su vez se conecta con la cultura de la participación entendida esta como la capacidad de los usuarios y las audiencias para reformular los procesos de producción y consumo cultural. En materia educativa el estado del conocimiento sobre este tipo de comunidades se ha concentrado más bien (de una forma muy limitada aún) en el análisis de comunidades de aprendizaje informal.

Actualmente existe una red mundial de comunidades que apuestan por aplicar la lógica del movimiento maker a las prácticas educativas y a las instituciones escolares de todos los niveles. En ese sentido, la investigación sobre los efectos e implicaciones de estas lógicas y éticas basadas en el DIY está aún por verse. En México, el trabajo que están realizando algunas comunidades de makers se ha llevado ya al terreno educativo sin conocer aún sus efectos y alcances reales.

La comunidad maker se encarga de empoderar a las personas por medio de la innovación y el conocimiento abierto, de tal manera que los afiliados tengan la posibilidad de recrear productos, y hacer realidad sus ideas sin recurrir a grandes inversiones. Todos son innovadores y todos comparten qué, cómo y por qué crean. En este sentido este movimiento 
da cuenta de una de las tendencias que está redefiniendo la relación de la sociedad con la tecnología: las innovaciones tecnológicas ya no son resultado exclusivamente del trabajo de grandes fabricantes y compañías multinacionales.

En el marco de la Estrategia Digital Nacional del gobierno de la República mexicana se lanzó en 2013 un programa de alcance nacional que buscó impulsar el desarrollo de proyectos de innovación y emprendimiento mediante el fomento de espacios de mejoramiento comunitario. En este esfuerzo por impulsar el desarrollo del emprendimiento se ofrecieron becas a proyectos de innovación con base tecnológica y mediática. En este contexto, han surgido voces que se ven tentadas a comparar el escenario incipiente de cultura digital, creativa y de la participación que está ocurriendo en Guadalajara para equipararla con la de Silicon Valley; esto se debe en buena medida al proyecto nacional para desarrollar en Guadalajara la Ciudad Creativa Digital (aún en construcción). En este marco, se han desarrollado en todo el país proyectos independientes para la creación de espacios físicos (makerspaces) y comunidades centradas en la creación y en el DIY.

Repensar las implicaciones que tienen estas comunidades en los alfabetismos a partir de la agencia de las personas no es algo totalmente nuevo, pero sí lo es la construcción de este objeto de estudio en función de un análisis comparativo que se preocupa por las prácticas de participación de los makers a partir de una reconfiguración de las maneras en que se consume, produce y circula información y conocimientos. $\mathrm{O}$, dicho de otro modo, investigar las culturas de la participación de comunidades de afinidad implica indagar sobre los "contratos participativos" que se están reconfigurando en un escenario de la comunicación y el aprendizaje donde predomina la interactividad, la autocomunicación de masas, la transmedialidad, la inteligencia colectiva y otras múltiples convergencias culturales y tecnológicas.

\section{Ethos DIY y cultura maker}

Considerar al DIY como un movimiento internacional solo es posible a partir de la Segunda Guerra Mundial, cuando, si bien el concepto no existía, la práctica generalizada de realizar mejoras y fabricar objetos fue algo necesario en muchas partes del mundo (Hallaq, 2010). Para la 
economía de los países protagonistas del conflicto fue indispensable que sus ciudadanos tomaran en sus manos la responsabilidad de reconstruir las ciudades y los hogares (principalmente en el caso de las naciones del Eje) y que impulsaran el desarrollo de la manufactura y las fábricas (sobre todo en el caso de los Aliados).

La evolución de la noción Hágalo usted mismo ha obedecido especialmente a que los referentes empíricos sobre los que se aplica (y a través de los cuales se puede observar y conocer) se han ido modificando con el paso del tiempo. La tecnología (en su sentido más amplio) relacionada con la técnica y la habilidad de las personas para el dominio y empleo de herramientas, ha sido determinante en las cosas que se pueden hacer una vez que se tiene acceso a ella, es decir, a las maneras del hacer y a los conocimientos necesarios para operar, reconvertir, administrar y combinar los recursos y la materia prima. En este sentido, quien se proponga hacer una historia detallada del concepto DIY tendría que revisar también cómo es que la tecnología ha permitido que la gente no especializada utilice el conocimiento disponible para su propio beneficio, para satisfacer sus necesidades y preferencias o para el beneficio compartido y social.

Luego de la etapa en que el concepto DIY se relacionó casi exclusivamente con el mejoramiento de los hogares y los espacios familiares (de la primera década del siglo Xx hasta la década de 1950), el siguiente paso significativo fue el impulso que cobró en las décadas posteriores con los movimientos contraculturales impulsados por los hippies y los punks (Moran, 2010). Uno de los ideales que reflejan la influencia del DIY en el movimiento punk (y acaso también viceversa) fue la intención de cambiar la industria musical del momento por medio de la producción de música original (a través de sellos independientes) que no replicara las formulas establecidas del momento y que asumiera una crítica activa basada en la construcción de una identidad que se reflejaba en acciones como la utilización de formatos de baja resolución en la grabación (DIY Lo-Fi) y la producción de la vestimenta (Spencer, 2005). Se llegó a afirmar incluso que para ser realmente un buen representante del movimiento punk habría que ser capaz de elaborar las prendas de vestir propias (Spencer, 2005).

En este sentido, el significado del concepto DIY se ha relacionado directamente con movimientos contraculturales que han representado algún tipo de resistencia al sistema dominante y que veían en su agencia un motor de cambio real y posible (Giddens, 1984). En esta lógica se han 
distinguido tres sentidos del DIY que es preciso recuperar para la construcción de un significado unificado que conviva tanto con las primeras significaciones como con las más recientes. El Hágalo usted mismo se entiende a partir de: a) la construcción de espacios propios, b) la creación de medios de comunicación (o expresión) alternativos, y c) el performance (entendido como acción directa y concreta sobre objetos, procesos o significados).

A partir de esta clasificación, entiendo el ethos DIY a partir de las "estructuras del sentir" de Williams (1983) en virtud de que las prácticas y la producción autogestionada de los prosumidores es visible en términos de actitudes, sentimientos, valores y lógicas compartidas por los miembros de comunidades creativas, y que dan forma no solo a su hacer colectivo sino también a una variedad de significados sobre su posición frente a los medios de comunicación masiva, las fuerzas de la economía capitalista, la educación institucionalizada, las formas de gobierno y otros elementos cruciales para entender el momento actual de la sociedad.

El ethos DIY tiene que ver más con las prácticas realizadas por las comunidades que con las características de los sujetos que integran esas comunidades. Este conjunto de actitudes y valores es visible en las prácticas que las comunidades realizan con la tecnología disponible y en el modo en que estas prácticas inciden en las determinaciones sociales a las que influencian, como la educación, la organización política, la construcción identitaria, el arte o la creación cultural.

Una de las cuestiones más interesantes en lo que se refiere a las actitudes y valores del ethos DIY es aquella que se refiere a la voluntad para encontrar y proponer nuevos usos a la tecnología y a los contenidos disponibles en los medios. Lo que implicaría un tipo de participación activa centrada en la creación que toma como inspiración el trabajo de otros y los productos de la cultura mediatizada y tecnológica.

El ethos DIY como un conjunto de valores, sentimientos y actitudes presentes en la actualidad ha tomado como referencia fundamental el impulso tecnológico y cultural de la ética hacker (Himanen, 2011) y la producción de software libre (Stallman, 2004), dado que estas lógicas (basadas en la libertad del hacer, en un sentido crítico y en la apertura para intercambiar conocimiento) son en buena medida responsables de la obtención de conocimientos y herramientas con las cuales participar activamente en la creación de contenidos y productos que resultan más accesibles a diferencia de otras épocas. 
El ethos DIY hace visible que estamos en presencia de una reconfiguración de la relación trabajo-ocio en la que las fronteras son cada vez más difusas y las lógicas de producción apuntan a un pensar con las manos, donde el disfrute de una práctica conduciría a la constante repetición de la misma y en consecuencia al dominio de dicha actividad. En este sentido los procesos de aprendizaje se ven necesariamente influidos y, en algunos casos, las prácticas del hágalo usted mismo tienen el objetivo de no solo influir sino también de reconvertir las relaciones de poder entre el conocimiento, el maestro y el educando. En este escenario, los procesos de comunicación y las lógicas transmediales tienen mucha injerencia. 


\section{Conclusiones}

Hablar de educación implica un tránsito constante entre las aspiraciones y deseos y las prácticas y experiencias. La educación entendida desde la escolarización implica un conjunto de procesos organizados cuya finalidad consiste en que los sujetos compartan y adquieran habilidades y conocimientos que han sido cultural y socialmente definidos y aceptados (Pérez, 2014). Bajo esta lógica, educación y escolaridad son dos aspectos fundamentales de una misma dimensión que tienen como fundamento la intencionalidad de enseñar. Pero la enseñanza es apenas una de las dimensiones que se ponen en juego en el proceso educativo, porque sin aprendizaje la enseñanza pierde su sentido y objetivos.

Los aprendizajes y alfabetismos más significativos no siempre se originan a partir de dinámicas institucionalizadas formales, sino que también ocurren como resultado de procesos autónomos, informales e invisibles casi siempre en el seno de comunidades y colectivos de la más diversa índole. En este sentido, no se trata de condenar la educación formal ni a la escuela, sino más bien de comprender sus lógicas tradicionales de operación y todo lo que pasa por fuera de ella. En medio de estas condiciones, la capacidad de agencia de los sujetos mediatizados se presenta como una situación que reconfigura el entorno comunicativo interactivo por medio de la participación activa, lo cual exacerba lo que podría pensarse como una crisis de los sistemas de educación escolarizados en función de un desfase con: a) los nuevos sistemas productivos y sociocognitivos, y b) la aparición de entidades colectivas que producen y circulan información y conocimiento sin necesidad de estar insertos en una institución educativa (Brunner, 2001). Es importante reconocer que esta crisis no es económica, sino que más bien está centrada en las discrepancias visibles entre la legitimidad institucional y la capacidad y posibilidad de los sujetos de acceder y construir conocimientos sin necesidad de grados o formación académica.

Transitar de las alfabetizaciones a los alfabetismos implica reconocer que una buena cantidad de información se transforma en conocimiento en 
el seno de las más diversas prácticas comunicativas y sociales. Es en este sentido que los alfabetismos transmediales cobran relevancia en cuanto dan cuenta de un tipo de aprendizaje que se caracteriza principalmente por los entornos de comunicación transmediáticos en los que el flujo de mensajes va de muchos a muchos y donde la participación de las personas es indispensable para la resignificación y la creación. 


\section{Referencias}

Alper, M., \& Herr-Stephenson, R. (2013). Transmedia Play: Literacy Across Media. Journal of Media Literacy Education, 5(2), 366-369. Disponible en https://bit.ly/2BJiais

Anderson, C. (2012). Makers: The new industrial revolution (Vol. 1). Estados Unidos: Crown Publishing Group.

Brunner, J. J. (2001). Globalización y el futuro de la educación: tendencias, desafíos, estrategias. En Análisis de prospectivas de la educación en la región de América Latina y el Caribe (pp. 47-89). Chile: UNESCO. Disponible en https://bit.ly/2LDoXi3

Castells, M. (2010). Comunicación y poder. Barcelona, España: Alianza. Dena, C. (2009). Transmedia practice: Theorising the practice of expressing a fictional world across distinct media and environments. Sidney, Australia: School of Letters, Art and MediaDepartment of Media and Communications Digital Cultures Program, University of Sydney.

Dussel, I. (2010). Aprender y enseñar en la cultura digital. Buenos Aires, Argentina: Fundación Santillana.

Estalella, A. (s. f.). Etnografías de lo digital [archivo PDF]. Recuperado de https://bit.ly/2QTdwJ8

Ferrés i Prats, J., \& Piscitelli, A. (2012). La competencia mediática: propuesta articulada de dimensiones e indicadores. Comunicar. Revista Científica de Comunicación y Educación, 19(38), 75-82. doi: http://dx.doi. org/10.3916/C38-2012-02-08

Gee, J. P. (2015). Literacy and Education. New York, NY: Routledge. Giddens, A. (1984). The constitution of society. Outline of the Theory of Structuration. Cambrige, MA: Polity Press.

Hallaq, T. (2010). DIY Media: Creating, Sharing and Learning with New Technologies. JMLE, 607, 41-55. Recuperado de https://bit.ly/2EY15Fw Hatch, M. (2014). The Maker Movemento Manifesto: Rules for Innovation in the New World of Crafters, Hackers, and Tinkerers. Nueva York, NY: McGraw-Hill. 
Himanen, P. (2011). La ética del hacker y el espiritu de la era de la información [Creative Commons]. Barcelona, España: E-book.

Ibrus, I., \& Ojamaa, M. (2014). What Is the Cultural Function and Value of European Transmedia Independents? International Journal of Communication, 8, 2283-2300. Recuperado de https://bit.ly/2TeH6W3 Jenkins, H. (2008). Convergence Culture. La cultura de la convergencia de los medios de comunicación. Barcelona, España: Paidós.

Jenkins, H. (2010). Confronting the Challenges of Participatory Culture: Media Education for the 21 Century. Cambridge, MA: MIT Press.

Kinder, M. (1980). The power of Adaptation in "Apocalypse Now". University of California Press, 33(2), 12-20. Disponible en https://bit.ly/2CErLca Lankshear, C., \& M. Knobel (2006). New Literacies: Everyday Practices and Classroom Learning. Nueva York, NY: Open University Press.

Libow Martinez, S., \& Stager, G. S. (2013). Invent to learn: Making, tinkering, and engineering in the classroom. Retrieved from http://www.amazon. ca/Invent-Learn-Tinkering-Engineering-Classroom/dp/0989151107 Livingstone, S. (2004). Media literacy and the challenge of new information and communication Technologies. The Communication Review, 1(7), 3-14. Recuperado de https://bit.ly/2Tf2b2U

Livingstone, S. (2008). Taking risky opportunities in youthful content creation: teenagers' use of social networking sites for intimacy, privacy and self-expression. New \& Media Society, 10(3), 393-411. Disponible en http://eprints.lse.ac.uk/1017

Moran, I. P. (2010). Punk: The Do-It-Yourself Subculture. Social Sciences Journal, 10(1), 58-65. Recuperado de https://bit.ly/2TdVbTN [http:// repository.wcsu.edu/ssj].

Orozco, G. (2011). La condición comunicacional contemporánea. Desafíos latinoamericanos de la investigación de las interacciones en la sociedad red. En N. Jacks y A. Marroquín (Eds.), Análisis de recepción en América Latina: Un recuento histórico con perspectivas al futuro (pp. 377-408). Quito, Ecuador: CIESPAL. Disponible en https://bit.ly/2MeN1ID Orozco, G. (2014). Mexican research no TV: a tradition framed by a powerful quasi-monopolistic TV system. En M. Alvarado, M. Buonanno, H. Grey, \& T. Miller (Eds.), The SAGE Handbook of Television Studies (pp. 105-116). Los Angeles, CA: SAGE. 
Pérez, V. (2014). De la calle a las aulas: el reto educativo de los nuevos alfabetismos digitales [archivo PDF]. Guadalajara, México: Universidad de Guadalajara, Centro Universitario de Ciencias Sociales. Disponible en https://bit.ly/2RhWSCt

Raybourn, E. M. (2014). A new paradigm for serious games: Transmedia learning for more effective training and education. Journal of Computational Science, 5(3), 471-481. doi: http://doi.org/10.1016/j.jocs.2013.08.005 Raymond, E. (2001). Cómo convertirse en hacker. Recuperado de https://bit.ly/2UtF4Sf

Rodrigues, P., \& Bidarra, J. (2014). Transmedia Storytelling and the Creation of a Converging Space of Educational Practices. International Journal of Emerging Technologies in Learning, 6, 42-49. Disponible en https:// bit.ly/2CDGSm5

Roig Telo, A. (2011). Trabajo colaborativo en la producción cultural y el entretenimiento [archivo PDF]. Barcelona, España: Editorial UOC. Recuperado de https://bit.ly/2rXaYLd

Scolari, C. (2008). This Is The End. Las interminables discusiones sobre el fin de la televisión. La Trama de La Comunicación, 13, 13-25. Recuperado de https://bit.ly/2CEcRmg Scolari, C. A. (2016). Alfabetismo Transmedia. Estrategias de aprendizaje informal y competencias mediáticas en la nueva ecología de la comunicación. TELOS, Revista de Pensamiento sobre Comunicación, Tecnología y Sociedad, (103), 13-23. Recuperado de https://bit.ly/2QZEqLq

Spencer, A. (2005). DIY: the rise of lo-fi culture. Londres, Reino Unido: Marion Boyars.

Stallman, R. M. (2004). Software libre para una sociedad libre [archivo PDF]. Madrid, España: Traficantes de Sueños. Disponible en https:// bit.ly/245o6cS

Wenger, E. C., McDermott, R., \& Snyder, W. M. (2002). Cultivating Communities of Practice. Cultivating Communities of Practice. Massachusetts: Harvard. doi: http://doi.org/10.1016/j.jchas.2013.03.426.

Williams, R. (1983). Keywords: A Vocabulary of Culture and Society. Londres: Fontana. 
